Fecha de recepción: febrero 2010 Fecha de aceptación: marzo 2010 Versión final: agosto 2010

\section{Comunicadores corporativos: desafíos de una formación profesional por competencias en la era global}

\author{
Claudia Gil Cubillos ${ }^{(*)}$
}

\begin{abstract}
Resumen: A lo largo de los años, la función del Director de Comunicación se ha ido consolidando en las organizaciones determinando a la vez una gestión profesional cada vez más compleja. En este contexto, dominado por la cultura virtual, las organizaciones adquieren una mayor visibilidad frente al entorno y, por lo tanto, están más conscientes y sensibles respecto de la importancia de gestionar la comunicación y las relaciones como herramientas estratégicas que agregan valor al negocio, no sólo fortaleciendo el posicionamiento en el mercado sino también como pieza clave para fidelizar y comprometer a sus públicos de interés (stakeholders) con los propósitos de la organización. Así, el perfil profesional en términos de las competencias que debe manejar quien asuma esa responsabilidad o trabaje en planes estratégicos de comunicación se vuelve cada vez más exigente. El debate se orienta a reflexionar respecto de redefinición de este perfil, para dar respuesta a un mercado organizacional que demanda comunicadores con rasgos más humanos y éticos, centrados en competencias cognitivas y técnicas sobre la disciplina.
\end{abstract}

Palabras claves: Comunicación Corporativa - Comunicación Empresaria - Comunicación Institucional - comunicación organizacional - Comunicadores - Dircom - Director de Comunicación - educación superior - formación profesional por competencias - Imagen Corporativa - Relaciones Públicas - Reputación Corporativa.

[Resúmenes en inglés y portugués y currículum en las páginas 58-59]

Dinamismo, competitividad, innovación y desarrollo acelerado de las nuevas tecnologías de información y comunicación, se constituyen en los principales ejes que orientan, mueven y dominan el mercado mundial en la era de la globalización.

Este nuevo escenario, caracterizado por los continuos y sucesivos cambios en los sistemas productivos, financieros, en la tecnología y la ciencia, demanda que las Instituciones de Educación Superior orienten sus propósitos educativos a la formación de profesionales integralmente desarrollados, individuos creativos-generativos, con habilidades para enfrentar los desafíos emergentes de la globalización y para participar en forma creativa e innovadora en la solución de problemas sociales y productivos (Parra, 2006, p. 3).

En coherencia con el contexto global y con fin de dar respuesta a las necesidades y tendencias del mercado laboral, las entidades de Educación Superior desde fines de los años 90 han orientado su proceso formativo hacia un modelo educativo basado en competencias, es decir en el desarrollo constructivo de habilidades, conocimientos y actitudes que permitan a los futuros profesionales insertarse adecuadamente en la estructura laboral y adaptarse a los cambios so- 
ciales (Marín, 2003, p. 19).

A partir de este nuevo enfoque, la educación se desarrolla en forma integral, se centra en el aprendizaje, el estudiante adquiere un rol activo-participativo en el aula y las prácticas vinculadas a la profesión se constituyen en un referente de la teoría entregada durante el proceso de formación. Esta nueva visión cambia significativamente el paradigma de la Educación Superior que por décadas concentró su quehacer en el desarrollo de conocimientos y destrezas en los estudiantes para ejercer una determinada profesión o disciplina, cumpliendo éste un rol pasivo-receptivo frente a un docente "dueño del saber".

Pero ¿qué gatilla este cambio en el modelo educativo de formación profesional? Simplemente, los efectos y desafíos de una economía globalizada y la estructuración de un mercado altamente competitivo, cambiante y exigente.

En un contexto de importantes transformaciones en el proceso productivo y de cambios tecnológicos en la organización social del trabajo, la formación profesional asume un papel fundamental como instrumento eficaz para facilitar el ajuste de la calificación de los recursos humanos a los requisitos del nuevo contexto (Vara y Guzmán, 2007).

Desde esta perspectiva, se modela la discusión internacional en materia de Educación Superior, orientada a reformular los procesos educativos de formación profesional con miras a establecer una relación virtuosa entre el sistema educativo y el productivo que promueva una inserción laboral exitosa.

Diversas entidades internacionales desarrollaron, desde mediados de la década del 90, una mirada proactiva y reflexiva frente a las transformaciones que requería la política educativa y los modelos curriculares en la Educación Superior para ajustarse a las necesidades de los nuevos tiempos. En 1995, el Banco Mundial entregó las primeras pautas sobre la materia y la Organización de las Naciones Unidas para la Educación, la Ciencia y la Cultura (UNESCO) publicó el documento "Cambio y Desarrollo de la Educación Superior", planteando los primeros desafíos en esta temática.

Dos años más tarde, la UNESCO se constituye en el referente sobre la visión educativa en el Siglo XXI con el Informe Delors ${ }^{1}$, que da origen a la Primera Conferencia Mundial de Educación Superior, realizada en París en octubre de 1998 y en la que participaron más de 180 naciones. Los acuerdos logrados en dicha Cumbre se plasmaron en la Declaración Mundial sobre Educación Superior en el Siglo XXI: Visión y Acción.

La Declaración Mundial de UNESCO establece las directrices para enfrentar los nuevos desafíos respecto de la enseñanza superior:

Las instituciones deberían asumir un nuevo modelo que responda a las profundas transformaciones en la enseñanza superior, un modelo que responda a las profundas transformaciones propias de la sociedad contemporánea y que considere el papel fundamental que tiene el conocimiento en esta era, y por supuesto el rol que las universidades cobran en este nuevo contexto histórico (González, 2007, p. 2).

Desde esta perspectiva el modelo educativo: 
...debería estar centrado en el estudiante, lo cual exige en la mayor parte de los países reformas en profundidad y una política de ampliación del acceso para acoger a categorías de personas cada vez más diversas, así como una renovación de contenidos, métodos, prácticas y medios de transmisión del saber, que han de basarse en nuevos tipos de vínculos y de colaboración con la comunidad y con los más amplios sectores de la sociedad (UNESCO, 1998).

Estos lineamientos se complementan con la Declaración de Bolonia de 1999, destinada a reformar el sistema educativo europeo bajo los principios de la calidad, movilidad, diversidad y competitividad, sobre la base del cambio de los métodos de enseñanza-aprendizaje. Ésta focaliza el quehacer hacia una atención más personalizada del estudiante, potenciando habilidades y destrezas que permitan su interacción fluida entre la formación y el trabajo.

Las intenciones de Bolonia se concretan a través del Proyecto Tuning, iniciativa destinada a reflexionar sobre la convergencia en términos de estructura y contenidos curriculares en las instituciones de Educación Superior, involucrando como actores claves de la discusión a más de 100 universidades integrantes de países de la Unión Europea, graduados y empresarios. Esta experiencia ha sido traspasada exitosamente a América Latina, dando vida al Proyecto TuningAmérica Latina.

El año 2000, el Banco Interamericano del Desarrollo (BID) plantea una propuesta estratégica para Latinoamérica, contenida en el documento La Educación Superior en América Latina y el Caribe: Estrategias, sumándose como un referente que entrega pautas respecto del rol de la Educación Superior y el modelo educativo a diseñar de acuerdo a la realidad de la Región. En la actualidad, es posible afirmar que el modelo de enseñanza-aprendizaje basado en competencias, se constituye en un enfoque validado de aplicación internacional y en el eje del quehacer de las entidades de Educación Superior, al articular la formación profesional en función de las demandas requeridas por el mercado laboral.

Si bien naciones como Estados Unidos, Canadá, Australia, Reino Unido y España, fueron las precursoras de este modelo de educación, esta dinámica se abrió también a Latinoamérica. Países como Argentina, Colombia, Chile, México, entre otros, se han sumado a los nuevos parámetros de formación y aparecen como pioneros de distintos procesos de modernización formativa vinculados al desarrollo de competencias profesionales, afianzándose así como un mecanismo coherente y pertinente para abordar la relación entre la formación y el empleo.

No obstante, resulta importante enfatizar que la incorporación del modelo ha implicado desafíos para las Instituciones de Educación Superior en términos de acciones y procedimientos que permitan su aplicación sistemática en sintonía con los fines esperados:

1. Diseñar perfiles profesionales mediante la identificación de las competencias requeridas en los diversos sectores de producción laboral.

2. Construir ofertas formativas en su sentido más amplio e instrumentalizar métodos y procesos de reconocimiento de las competencias profesionales.

3. Construir currículos de formación profesional relacionadas con las necesidades profesionales previstas para cada uno de los sectores del mercado laboral. (Vara y Guzmán, 2007).

La articulación de cada una de estas iniciativas ha sido una brújula en el actuar de las entidades de enseñanza superior en la última década. 


\section{Enfoque del Modelo Educativo por Competencias}

En el actual contexto de globalización, las organizaciones se enfrentan a un cambiante entorno político, social y económico, que repercute indudablemente en la relación y forma en que empresas e instituciones abordan la gestión de personas.

Uno de los cambios más significativos que podemos advertir tiene que ver con la creciente disminución del vínculo emocional entre la empresa y los trabajadores (...) como una consecuencia casi espontánea de la volatilidad de las organizaciones en esta nueva economía (fusiones, adquisiciones, reestructuraciones) (...) Si las empresas no son capaces de garantizar su permanencia en el mediano y largo plazo, menos podrían asegurar la permanencia de sus trabajadores (...) En este escenario se redefinen los compromisos e identificación de las personas con las organizaciones (...) aparece un mayor individualismo por parte de las personas y un fuerte énfasis en un marcado desempeño por parte de la empresa (cultura al mérito) y de la retribución asociada (Volenski, 2006, p. 4).

Se aprecia así una transformación en el perfil de profesionales que demanda el mercado laboral. Se pasa de un modelo tradicional laboral de dependencia y subordinación a una relación basada en la confianza y compromisos mutuos, lo que trae aparejado una redefinición de la carrera laboral y profesional: "cada trabajador se hace cargo de su proyecto profesional y toma el control de su propia carrera. Se reinventa permanentemente... debe ser capaz de enfrentar desafíos de alta complejidad y desenvolverse en entornos cambiantes e hipercompetitivos" (Volenski, 2006, p.5). A esta realidad se suma un sistema de medición del desempeño laboral aplicado por una empresa u organización basado en las competencias del colaborador para efectuar un trabajo o labor: aspectos cognitivos (acerca de los que sabe); habilidades y destrezas que explican sus conductas (acerca de lo que hace), y las actitudes y valores que presenta el individuo (acerca de lo que se es) (Allen, 2006, p.61).

Desde esta perspectiva es factible afirmar que el cambio en las necesidades profesionales experimentadas por el entorno laboral sustenta el actual modelo educativo por competencias, incorporado progresivamente por las entidades de Educación Superior a nivel mundial. En el caso de Chile es precisamente esa necesidad de relacionar en forma efectiva la educación con el mundo del trabajo lo que ha impulsado cada vez más a universidades e institutos a ofrecer carreras o programas académicos que promueven una formación en esta línea.

De acuerdo a lo expuesto, ¿qué se entiende por competencia en el mundo académico? ¿Qué componentes estructuran un modelo educativo curricular con estas características? ¿Cómo se relaciona el modelo educativo por competencias con las necesidades del mundo laboral? Diversas son las definiciones y reflexiones que presenta la bibliografía disponible sobre educación y gestión de recursos humanos para dar respuesta a estas preguntas.

La Organización para la Cooperación y Desarrollo Económico a través de su Proyecto DeSeCo (Definition and Selection of Competencies) define el término competencia como:

Capacidad de responder a demandas complejas y llevar a cabo tareas diversas en forma adecuada. Supone una combinación de habilidades prácticas, conocimiento, motivación, valores éticos, actitudes, emociones y otros componentes sociales y comportamientos que se movilizan conjuntamente para lograr una acción eficaz. 
Para José Joaquín Brunner, destacado sociólogo chileno y experto en educación, el concepto competencia se refiere al conjunto de capacidades que incluye conocimientos, actitudes, habilidades y destrezas que una persona logra mediante procesos de aprendizaje y que se manifiestan en su desempeño en situaciones y contextos diversos, y hacen eficaces a las personas en una determinada situación para resolver problemas. (Brunner, 2009).

Una definición más asociada al mundo organizacional y que complementa las anteriores, es la desarrollada por los autores Spencer \& Spencer: característica subyacente a un individuo que está causalmente relacionada con un estándar de efectividad y/o con una performance superior en un trabajo o situación. Característica subyacente significa que corresponde a una parte profunda de la personalidad; causal relacionada es entendida como comportamiento; y estándar de efectividad como la predicción de quien hace algo bien o no (Alles, 2006, p.59).

Si bien cada definición pone énfasis en conceptos distintos, el denominador común es la necesidad de una formación y desempeño integral de una persona sobre la base de sus conocimientos, habilidades y actitudes al desenvolverse y enfrentar situaciones propias del entorno. Respecto de la clasificación de las competencias, diversos autores coinciden en las siguientes:

- Competencias Básicas: capacidades intelectuales indispensables para el aprendizaje de una profesión de carácter cognitivo, técnico y metodológico.

- Competencias Genéricas: aquellas comunes a varias profesiones por ejemplo, capacidad de trabajo en equipo, habilidad para planificar y organizar, resolución de problemas, aprender a aprender.

- Competencias Específicas: se refiere a aspectos directamente relacionados con la profesión o la carrera que propician el desempeño específico en un campo de aplicación concreto.

Desde una perspectiva educativa, la exitosa implementación de un modelo por competencias requiere que toda entidad de Educación Superior defina a partir de su propio modelo pedagógico y oferta de carreras, qué enfoque y tipo de competencias acentuará en sus estudiantes. Lo anterior, con el propósito de estructurar el proceso de enseñanza-aprendizaje sobre la base de los cuatro pilares esenciales en educación establecidos por UNESCO: Aprender a Conocer, Aprender a Hacer, Aprender a Vivir Juntos, Aprender a Ser. Pero, ¿cómo define UNESCO cada uno de estos pilares?

Aprender a Conocer combinando una cultura general suficientemente amplia con la posibilidad de profundizar los conocimientos en un pequeño número de materias. Supone además: aprender a aprender para poder aprovechar las posibilidades que ofrece la educación a lo largo de la vida.

Aprender a Hacer a fin de adquirir no sólo una calificación profesional sino, más generalmente, una competencia que capacite al individuo para hacer frente a gran número de situaciones y a trabajar en equipo. También aprender a hacer en el marco de las distintas experiencias sociales o de trabajo que se ofrecen a los jóvenes y adolescentes, bien espontáneamente a causa del contexto social o nacional gracias al desarrollo de la enseñanza por alternancia. 
Aprender a Vivir Juntos desarrollando la comprensión del otro y la percepción de las formas de interdependencia -realizar proyectos comunes y prepararse para tratar los conflictos- respetando los valores de pluralismo, comprensión mutua y paz.

Aprender a Ser para que florezca mejor la propia personalidad y se esté en condiciones de obrar con creciente capacidad de autonomía, de juicio y de responsabilidad personal. Con tal fin, no menospreciar en la educación ninguna de las posibilidades de cada individuo: memoria, razonamiento, sentido estético, capacidades físicas, aptitud para comunicar.

Fuente: Informe a la UNESCO de la Comisión Internacional sobre la Educación para el Siglo XXI, presidida por Jacques Delors (1998)

A partir de estos lineamientos, la estructuración del currículo basado en competencias requiere previamente de una definición del perfil de egreso del estudiante en sintonía con el perfil profesional que buscan las empresas e instituciones en el nuevo escenario global y que va más allá de lo académico y de la experiencia adquirida por una persona.

Competencias tales como iniciativa, autogestión, trabajo en equipo, planificación y flexibilidad para adecuarse a diversos contextos, son frecuentemente mencionadas por directivos de las áreas de recursos humanos al momento de describir las características de los profesionales que demandan las organizaciones en el siglo XXI.

Desde esta visión más integral, se entiende que la formación del futuro profesional debe estar orientada hacia la autogestión de su propio aprendizaje a través de la interacción con la información, asumiendo una actitud crítica, creativa y reflexiva que le permita ir aplicando lo que aprende en el entorno cotidiano (Parra, 2006, p.6).

De esta manera, el desafío para quienes ejercemos cargos directivos en entidades de Educación Superior, es generar un diseño curricular en función del quehacer de la institución -tipo de programas académicos que ofrece- considerando siempre los diversos contextos profesionales a los que se enfrentarán los egresados. Esto significa no sólo incorporar una dimensión de aplicación práctica de conocimientos y habilidades, sino verificar que dicha práctica coincida con las necesidades estratégicas de las organizaciones. En este sentido, toda innovación curricular necesariamente debería ir acompañada por una medición de resultados de desempeño en los diferentes niveles de una organización para establecer así una conexión entre la formación profesional entregada y los logros alcanzados en el contexto organizacional (Mertens, 2000, p.60).

\section{Aplicación del Modelo: competencias claves de un comunicador corporativo}

La globalización ha generado una sociedad más activa, que se organiza en torno a causas comunes, que vela por sus derechos y establece sus demandas de acuerdo a sus necesidades e intereses. En este contexto, dominado por la cultura virtual, las organizaciones adquieren una mayor visibilidad frente al entorno y, por lo tanto, están más conscientes y sensibles respecto de la importancia de gestionar la comunicación y las relaciones como herramientas estratégicas que agregan valor al negocio, en términos no sólo de fortalecer el posicionamiento de una entidad frente a sus competidores, sino también como pieza clave para fidelizar y comprometer a sus 
públicos de interés (stakeholders) con los propósitos de la organización.

Crear oportunidades para comunicar con eficacia se constituye una necesidad para cualquier organización moderna. Las empresas no son organizaciones aisladas, sino que están sujetas a las influencias y a los mensajes del entorno, y, a su vez, proyectan sobre éste una imagen que es percibida por todos sus clientes. Esta imagen no es otra cosa que el concepto que los destinatarios tienen después de recibir los mensajes. De ahí que el desarrollo de una correcta política de comunicación implique beneficios añadidos a las empresas. Los directivos encargados de tomar las decisiones finales deben ser cada día más conscientes que la comunicación ha dejado de ser un lujo para convertirse en una necesidad ineludible (Seijo, 2007, p.3).

Estudios e investigaciones recientes evidencian que el éxito de una empresa y/o la agregación de valor, radica fundamentalmente en el capital humano y en la gestión de relaciones, quedando éste supeditado en gran medida a la eficiente y proactiva gestión de activos intangibles, entre ellos aspectos como la comunicación y reputación (Volenski, 2006, p.8).

A lo anterior se suma el rol cada vez más relevante que adquieren las políticas de transparencia y sostenibilidad en el escenario global. Actualmente, existe una imperante necesidad de incorporar la ética empresarial como una variable dentro de la estrategia de la organización. En este sentido un esquema de rendición de cuentas al interior de la empresa resulta un desafío fundamental para transformar y divulgar la Responsabilidad Social Empresarial (RSE) como un activo estratégico (Murria, 2006).

De ahí la necesidad que las organizaciones incorporen a un profesional- Comunicador Corporativo o Relacionador Público según como se le denomine a la profesión en cada país- cuyo aporte, precisamente, se oriente a definir la política comunicativa, a estructurar una estrategia de comunicación en función de los objetivos estratégicos de la organización y a gestionar la imagen corporativa de ésta.

A lo largo de los años, la función del Director de Comunicación se ha ido consolidando en las organizaciones. Según el comunicólogo Joan Costa "Las empresas necesitan la figura del Dircom, debido a que su trabajo es cada vez más complejo, ya que hay más frentes a los que dar respuesta, Se requiere de alguien que se ocupe de un asunto tan importante como es el de las comunicaciones" (Costa, 2009).

Respecto del perfil profesional, en términos de las competencias que debe manejar quien asuma esa responsabilidad o trabaje en planes estratégicos de comunicación, Costa enfatiza que además de ser un buen estratega en términos de planificación, implementación y evaluación de iniciativas comunicacionales, “(...) se requiere de personas con sentido de responsabilidad, capaces de establecer relaciones con la gente, que sean sociables, que posean carácter de liderazgo para dirigir a un equipo y con una visión global de las cosas" (Costa, 2009). Entidades como la European Association of Communication Directors (EACD) y la Association of Executive Search Consultants (AESC) han incentivado la discusión respecto de la identidad de los comunicadores empresariales en los nuevos tiempos. El debate se orienta a reflexionar respecto de la necesidad de redefinir su perfil para dar respuesta a un mercado organizacional que demanda comunicadores con rasgos más humanos y éticos que centrados en competencias cognitivas y técnicas sobre la disciplina.Esta visión hacia un nuevo perfil se explicaría por las características de la Nueva Economía. Las empresas e instituciones se enfrentan a permanentes cambios, desafíos y dilemas éticos y, en consecuencia, el profesional que gestiona las comunicaciones debe manejar en forma equilibrada sus propias virtudes y principios éticos con los intereses corpo- 
rativos.Lo anterior, revela la importancia de otorgar a los futuros Comunicadores Corporativos o Relacionadores Públicos una formación académica basada en un modelo por competencias, cuyo diseño curricular incorpore las actuales demandas laborales tales como flexibilidad, creatividad e innovación, sin descuidar la formación integral en los ámbitos humanos, profesionales y disciplinarios. A partir de esta visión, la educación basada en competencias enriquece y retroalimenta considerablemente el currículo y puede constituirse en una propuesta de formación profesional más actualizada y de mayor calidad (Huerta, 2000).

El desafío para quienes ejercemos la docencia y participamos directamente en el proceso formativo de Comunicadores Corporativos o Relacionadores Públicos, es garantizar que el modelo curricular integre contenidos y metodologías de aprendizaje que combinen teoría y práctica en función de las necesidades de la profesión y de las temáticas emergentes en la disciplina. Es decir, que articule conocimientos globales, profesionales y experiencias laborales sobre la base de los conceptos que guían el modelo educativo del Siglo XXI: Saber, Saber Hacer, Saber Convivir.

De esta manera, el perfil de egreso definido por la institución académica dará respuesta a los requerimientos del mundo organizacional en la disciplina: profesionales de excelencia, éticos en su actuar, polivalentes y multimediales, capaces de diseñar, implementar y evaluar estrategias de comunicación y con el manejo de competencias orientadas al liderazgo, al trabajo en equipo, a la resolución de problemas, a la adaptabilidad, a la gestión de relaciones de confianza con sus públicos de interés, entre otras, que les permitan enfrentar con éxito los retos de un escenario laboral altamente competitivo y dinámico.

No obstante, es importante considerar, como modo de controlar la flexibilidad y transferencia de competencias, la incorporación al currículo de períodos de prácticas modulares de los estudiantes durante su formación profesional (Scheele y Brunner, 2009, p.5).

En el caso de la formación de Comunicadores Corporativos o Relacionadores Públicos, resulta pertinente que el modelo pedagógico se estructure sobre la base de una relación sistemática con el medio laboral, a través de ejercicios académicos que involucren a los estudiantes con proyectos y dilemas comunicacionales propios de empresas o instituciones reales. De esta manera, se logrará contextualizar de manera óptima el aprendizaje teórico-práctico bajo el concepto metodológico "Aprender-Haciendo", permitiendo así monitorear, modelar y enfatizar las competencias más requeridas durante el proceso de formación profesional.

De esta manera, las Instituciones de Educación Superior y especialmente quienes dirigen programas académicos orientados a formar profesionales expertos en Comunicación Corporativa o Relaciones Públicas, deben plantearse el desafío de la educación como una responsabilidad y compromiso centrado en la calidad y la excelencia. Ello, unido al rigor de incorporar al mercado profesionales con los conocimientos teóricos y competencias laborales en comunicación corporativa en sintonía con las necesidades del entorno, pero por sobretodo, comunicadores con valores, principios y calidad humana para liderar la gestión comunicacional de empresas y organizaciones en un contexto altamente flexible y cambiante.

\section{Notas}

1 Informe "La Educación Encierra un Tesoro" (1998) solicitado por UNESCO a la Comisión Internacional para la Educación del Siglo XXI, presidida por Jacques Delors. Plantea que la educación debe estructurarse sobre la base de cuatro pilares: Aprender a Ser, Aprender a Cono- 
cer, Aprender a Hacer y Aprender a Vivir Juntos. El énfasis de la enseñanza no sólo debe estar en el conocimiento, sino en el sentido humano.

\section{Referencias Bibliográficas}

Alles, M. (2006). Dirección Estratégica de Recursos Humanos. Gestión por Competencias. $2^{\circ}$ Edición. Buenos Aires: Granica.

Brunner, J. (2009). Artículo Congreso Internacional Fortaleciendo las Competencias: Nuevas Estrategias, Nuevos Aprendizajes. Madrid. Disponible en: http://mt.educarchile.cl/MT/jjbrunner/archives/2009/08/congreso_intern.html

González, O. (2007). Desarrollo de competencias y enseñanza universitaria. Consensus, ene./dic. 2007, vol.12, no.1. Disponible en: http://revistas.concytec.gob.pe/scielo.php?script=sci_ arttext\&pid=S1680-38172007000100011\&lng=es\&nrm=iso

Huerta, J. (2000). Desarrollo Curricular por Competencias Profesionales Integrales. Revista de Educación. Nueva Época, $\mathrm{N}^{\circ} 13$. Abril-Junio 2000.

Marín, R. (2003). El Modelo Educativo de la Universidad Autónoma de Chihuahua. Elementos para su Construcción. Dirección Académica Universidad Autónoma de Chihuahua. México. Disponible en: http://comunidad.uach.mx/wgutierr/modeloeducativoUACH.pdf

Meters, L (2000). La Gestión por Competencia Laboral en la Empresa y la Formación Profesional. Organización de Estados Iberoamericanos para la Educación, la Ciencia y la Cultura. Disponible en: http://www.oei.es/oeivirt/fp/iberfop01.htm

Murray, R. (2006). Artículo La Transparencia Corporativa. Comunica RSE. Comunicación de Responsabilidad Social de la Empresa. Disponible en: http://www.comunicarseweb.com.ar/

OCDE (Organización para la Cooperación y Desarrollo Económico) (2005). Resumen Ejecutivo Proyecto DeSeCo (Definition and Selection of Competencies). Disponible en: http:// mt.educarchile.cl/MT/jjbrunner/archives/libros/Competencias/OECD2005.pdf

Parra, H. (2006). El Modelo Educativo por Competencias Centrado en el Aprendizaje y sus Aplicaciones en la Formación Integral del Estudiante Universitario. Documento Sexto Congreso Internacional: Retos y Expectativas de la Universidad. Universidad Autónoma de Chihuahua. México. Disponible en: http://www.congresoretosyexpectativas.udg.mx/Congreso\%206/Eje\%202/Ponencia_212.pdf

Linares, S. (2009). Entrevista a Joan Costa. “Las Empresas Necesitan al DIRECOM” ACAM en Breve. Universidad Católica de Santo Tomás de Murcia, España. Disponible en: http://enbreve.ucam.edu/index.php?option=com_content\&view=article\&id=329:joan-costa-las-empresas-necesitan-al-dircom\&catid=84:titulaciones\&Itemid $=201$

Seijo, C.(2007). Gerencia Ética de la Comunicaciones en las Organizaciones del Futuro. Revista Electrónica Psicología Científica. Disponible en: http://www.psicologiacientifica.com/bv/ psicologia-257-6-gerencia-etica-de-la-comunicacion-en-organizaciones-de-futuro.html-

Scheele, J; Brunner, J.(2009). Educación Terciaria y Mercado Laboral: Formación Profesional, Empleo y Empleabilidad. Revisión de la Literatura Internacional. Centro de Políticas Comparadas de Educación. Universidad Diego Portales, Chile. Disponible en: http://www.cpce.cl/es/ publicaciones/docman/cat_view/24-documentos-de-trabajo?start=5

UNESCO (1995). Documento "Cambio y Desarrollo de la Educación Superior".

UNESCO (1998).Declaración Mundial Sobre la Educación Superior en el Siglo XXI: Visión y 
Acción. Disponible en: http://www.unesco.org/education/educprog/wche/declaration_spa.htm Vara, A. Guzmán, C.(2007). La Formación Profesional y la Demanda Laboral. Disponible en: http://www.aristidesvara.com/investigaciones/administracion/demandalaboral/demandal. htm

Volenski, G. (2006). Trabajo y Gestión de las Personas en la Nueva Economía. Santiago de Chile: Universidad Adolfo Ibañez

\section{Bibliografía}

Banco Interamericano del Desarrollo (2000). La Educación Superior en América Latina y el Caribe. Documento de Estrategia

Bravo, H (2007). Paper Competencias Proyecto Tuning-Europa/Tuning América Latina. Bogotá, Colombia.

Brunner, J. (2007). Educación Superior en Iberoamérica: Informe 2007. Santiago de Chile: Centro Universitario del Desarrollo (Cinda)

Declaración de Bolonia (1999). Disponible en: http://universidades.universia.es/fuentes-info/ documentos/bolonia.htm

Gonzalez, O (2007). Desarrollo de competencias y enseñanza universitaria. Consensus, ene./dic. 2007, vol.12, no.1, p.109-114. ISSN 1680-3817.

Ministerio de Educación de Chile (1998). El Impacto de la Globalización en la Educación Supe rior Chilena. División de Educación Superior, Santiago de Chile

Muñoz, K. (2009). Comunicación Estratégica. Universidad y Mediaciones, Reflexiones de una Docencia Especializada en la Profesión de Relaciones Públicas. Revista electrónica Razón y Palabra. $N^{\circ} 70$. México. Disponible en: www.razonypalabra.org.mx

Spencer, L. y Spencer, S. (1993). Competence at Work For Superior Performance. EE.UU.: John Wiley \& Sons Inc.

Tironi, E. Cavallo, A (2007). Comunicación Estratégica. Vivir en un Mundo de Señales. Madrid: Taurus.

UNESCO (1998). La Educación Superior Encierra un Tesoro. Informe de la Comisión Interna cional de Educación para el Siglo XXI. Informe Delors. Madrid: Santillana.

Universidad UNIACC (2009). Fundamentos del Currículo: Modelo UNIACC.

Yañez, E. (2003). Manual de Ética de las Relaciones Públicas. Dirección de Formación General. Santiago de Chile: DuocUC.

Summary: Throughout the years, the function of Communications Director has been consolidated in organizations increasing the complexity of professional management. In this scenario, dominated by virtual culture, organizations acquire a greater visibility and they become more conscious and sensitive towards communication management relevance. They also realized that organization relationships are strategic tools that add value to the business, not only fortifying their position in the market but also reinforcing stakeholder's commitment with the company. Thus, the professional profile in terms of the competitions that must handle the person who assumes that responsibility or works in strategic plans of communication becomes increasingly demanding. The debate is oriented to reflect about the redefinition of 
this profile, to give answer to an organizational market that demands communication experts with more human and ethical characteristics, focused in mental and technical competitions on the discipline.

Key Words: Communication experts - Director of Communication - Dircom - organizational communication - professional education by competitions - Public relations - superior education.

Resumo: Ao longo dos anos, a função do Diretor de Comunicação foi-se consolidando nas organizações determinando ao mesmo tempo uma gestão profissional cada vez mais complexa. Neste contexto, dominado pela cultura virtual, as organizações adquirem uma maior visibilidade frente ao meio e, portanto, estão mais conscientes e sensíveis à importância de gerir a comunicação e as relações como ferramentas estratégicas que agregam valor ao negócio, nao só fortalecendo o posicionamento no mercado senão também como peça fundamental para fidelizar e comprometer seus públicos de interesse (stakeholders) com os propósitos da organização. Assim, o perfil profissional em termos das concorrências que deve conhecer quem assuma essa responsabilidade ou trabalhe em planos estratégicos de comunicação, se volta cada vez mais exigente. $\mathrm{O}$ debate deve reflexionar a redefinição deste perfil, para dar resposta a um mercado das organizações que demandam comunicadores com características mais humanas e éticas, baseados em concorrências cognitivas e técnicas da disciplina.

Palavras chave: comunicação organizacional - Comunicadores - Dircom - Diretor de Comunicação - educação superior - formação profissional por competências - Relações públicas.

(*) Licenciada en Comunicación Social. Magíster en Comunicación Estratégica (Pontificia Universidad Católica de Chile). Periodista (Universidad Finis Terrae, Chile). [Ver CV completo en la página 152] 